\title{
"Internet+" Area Enterprise Information Security Research
}

\author{
Li Guang-hui \\ XingTai University, \\ Xingtai, Hebei 054000
}

\author{
Su Jing \\ XingTai University, \\ Xingtai, Hebei 054000
}

\author{
Ding Zhao-han \\ XingTai University, \\ Xingtai, Hebei 054000
}

\begin{abstract}
- 2015, in the government work report, "Internet +" has been formally put forward to determine the status of "Internet + " in national economic development. So-called "Internet +" refers to a new economic form, which is about technology and the Internet business integrate to achieve the goal of promoting business development in the "Internet + " era. The production and operation of enterprises can no longer follow the old path. Instead, give full play to the role of Internet technology in the allocation of production factors in the Internet technologies in the production business practice, thereby enhancing innovation capacity and market competitiveness. Although the "Internet +" era provides unprecedented opportunities for the development of the enterprise, but also brings a series of problems, including most major one, corporate information security. Therefore, under the "Internet +" era background, the research on corporate information security is necessary.
\end{abstract}

Keywords- Interne+, Enterprise, Information security

\section{INTRODUCTION}

"Internet + " era makes the Internet technology application to become more widespread in all kind of fields, greatly improving the speed of social development, but also makes the social productive activities increasingly inseparable from the Internet technology. People's dependence on Internet technology is getting stronger. While Internet technology has a great advantage in production activities, it also has disadvantages. One problem that people are most concerned about is the business information security issues. In "Internet + "era, if an enterprise information leakage incidents happen, it will impose a fatal blow. Therefore, during the development of Internet technology, we must attach importance to corporate information security issues. This article describes the" Internet + " era enterprise information security threats coming up with strategies to improve enterprise information security management system measures and improve enterprise information security technology to prevent system in the hope of enterprise information security management works in the future.

\section{Business information security threats in Internet + "era}

According to relevant survey data, in the tens of millions new emerging domestic APP in 2014, $86 \%$ of the APP security is vulnerable, while nearly 40 percent of APP are with viruses or illegal advertising. Most global companies have 
encountered over information security issues, economic losses caused by information security problems reached $\$ 445$ billion.

\subsection{Information safety and security problems in development of big data}

At this stage, the use of relatively high frequency database in practice is relational database, and big data is in unstructured data. This type of data is difficult to store relational database, which means that with a relational database, some information security technology is difficult to apply to large data management, giving corporate information security planted a serious security risk. At the same time, big data is quite different form previous data in, big data sources are relatively more open, difficult to effectively manage their business through traditional data management methods and brings data management confusion in corporate.

\subsection{DDoS and APT attacks threat confidential corporate information}

In general, the master program will be installed on a computer, the agent is installed on the remaining multiple computers, while master program and the agent can communicate within a specified period of time, the master program controls agent mainly via client / server technology, it will immediately initiate the target once the agent receives the order to attack. Compared with DDoS attacks, ATP attacks are more advanced .Before ATP attack, it will gather information, and the main target is to collect relevant information. In the process of gathering information, ATP attack would target their own vulnerabilities to collect, so an attacker can complete its own loopholes attack missions use to attack the target.

\subsection{Mobile security threats become new challenge to enterprise information security}

The emergence of the mobile terminal makes the business office efficiency has been greatly improved, employees can send and receive e-mail directly from the phone, share and transmit information through. However, the use of personal office equipment has a very big security risk for the attacker to provide a new way to steal corporate information. In 2014, eBay Web site user information was stolen, but the cause of this problem was that the staff logged in the account at the mobile terminal.

\subsection{Enterprise information security management loopholes}

First, there is "Breakpoints" in the enterprise information system. Companies will inevitably exist "break" in the information construction process, which is also the information flow interruption phenomenon that must be completed before the information transmitted by manual operation. The information system "break" the key point is the result of enterprise information security problems. Secondly, enterprises lacks effective information management and monitoring processes.

Enterprise information security management is a relatively strong integrated systems engineering, which includes both the institutional level management, but also includes management level. Therefore, the enterprises needs to cope with the information security threats under "Internet +" background and through the information security management system and information security technology two-pronged approach. 


\section{Countermeasure to improve the system of enterprise information safety management countermeasures}

\subsection{Hierarchical management for enterprise information}

Enterprise Information adopt hierarchical management style mainly includes three aspects. First, deal with the enterprise information management at different levels based on the importance of information and its sharing range. The classification is divided into several stages based on the specific actual business situation. Secondly, enterprises classified information should use hierarchical management device according to the confidentiality classification and the use of authority. Finally, to deal with companies involved secret hierarchical management personnel, enterprises should clearly define access to information for staff in various positions and the secret information processing method.

\subsection{Strengthen the management of the secret related personnel}

Enterprises secret related personnel management should include three stages. The first stage is the appointment management, training should be executed before the employees' onboarding so that employees understand the nature of the work and safety requirements. The second stage is on duty management, which should be done from two aspects of the work. On the one hand corporate should strengthen the professional ethics education to avoid the sale of corporate information from staff. On the other hand should strengthen security and technical education for employees to master the basic security technology. The third stage is undergo management.

\subsection{Strengthen computer security management}

Enterprise computer security management should include two aspects. First, purchase, installation, commissioning and maintenance must be carried out strictly in accordance with the provisions of the enterprise; second, business daily use of computers and related information must strictly comply with the regulations of corporation.

\subsection{Strengthen removable storage media management}

As the situation of mobile storage device abuse, corporation must establish mobile storage device management system in line with the actual situation of enterprise. Mobile storage device management system should clearly define the use range of removable storage devices, methods, content and other operations.

\section{Countermeasures to improve enterprise information security technology}

Institutional management is just a part of the corporate information security management, enterprises should strengthen the improvement of information security technology to solve business problems in the management of information security from a technical level.

\subsection{Terminal Access}

Terminal access includes two aspects. First, a mobile terminal equipment must be registered only after certification by corporate to connect to the internal network computer. Mobile terminal device can connect to the internal network with registration. Second, after registration, users are required to complete the user name and password authentication; third, access restrictions should be based employees' 
positions to limit their access to enterprise information level.

\subsection{Application control}

Applications should include four parts. First, equipment must be certified for export which is connected to the enterprise network when off the network terminal in order to truly ensure the security of corporate information; Second, after terminal equipment connect to the enterprise network, the use of terminal equipment for recreational activities should be prohibited, it should also prohibit the access to internet on multiple machines simultaneously; Third, monitor the use of information device under the premise of the enterprise according to national laws and regulations, and the monitoring logs should be preserved for future inspection; Forth, prohibit employees to use the corporate intranet access without regular site, to avoid information leakage

\subsection{Monitoring audit}

Monitoring audit system includes messaging, file copy, computer operation, data information and monitor the activities of saving in the form of logs. On the one hand you can determine whether the enterprise exist information disclosure issue by monitoring $\log$, on the other hand it can also track the source of corporate information disclosure by monitoring log. Those responsible be accountable.

\subsection{Virus prevention}

To ensure the security of corporate information, we must adopt effective measures to prevent the virus. First, improve the scanning frequency, regularly check your computer for viruses; second, to install professional anti-virus module in the server, and timely kill the virus; and third, it should set up a firewall, shielding the virus which will cause a computer from viruses interference.

\section{Conclusion}

In short, the "Internet + " era will significantly improve the innovation capacity of enterprises, thus improve business competitiveness in the market. However, the "Internet + " era will make corporate information security face serious challenges. Therefore, enterprises shall pay attention to information security issues under the "Internet + " background to get better development.

\section{Acknowledgment}

This research was financially supported by the Social Science Foundation of Hebei Province (Grant NO. HB15YJ009).

\section{References}

[1] Cheng Hong, Fan Hanbing and Xiao Yu, Quality and safety risk theoretical framework of effective governance Quality of Internet information security classification model based implementation and method, Management World, vol.12, pp.73-81, 2012.

[2] Chen Liru, Innovation of Enterprise Value Network Internet Economy,China Industrial Economy, vol.9, pp.82-94, 2013.

[3] Yang Huixin, Li Feng and Wu Weifeng, Border and strategic choice with Internet, China Industrial Economy, vol.11, pp.88-97, 2008. 